\title{
Measuring Adherence to ARVs among HIV-positive Adolescents in Cameroon: A Comparative Assessment of Self-report and Medication Possession Ratio Methods
}

Mbuwir Charlotte Bongfen ( $\sim$ charlotte.bongfen@yahoo.com )

Biaka University Institute Buea, Cameroon https://orcid.org/0000-0002-8517-6392

Kwasi Torpey

University of Ghana

John Ganle

University of Ghana

Ankomah Augustine

University of Ghana

\section{Research note}

Keywords: Adherence measurements, adolescents, self -report, medication possession ratio, viral load suppression

Posted Date: November 10th, 2020

DOl: https://doi.org/10.21203/rs.3.rs-101994/v1

License: (9) This work is licensed under a Creative Commons Attribution 4.0 International License.

Read Full License 


\section{Abstract}

\section{Objective}

Adherence to ARV medications has been shown to improve treatment outcomes in HIV positive patients. Given that ARV treatment is lifelong, adherence has become a critical issue as it may reduce over time. Measuring adherence is therefore imperative in programming. There are different methods of measuring adherence each with its advantages and disadvantages, depending on the context and the time. This study therefore compares two widely used adherence measurement scales in Cameroon, namely, the selfreport and the Medication Possession Ration (MPR) methods.

\section{Results}

The study shows that adherence level using the self-report technique is $82.9 \%$ while that of MPR was $73.4 \%$. When compared using the using Kappa statistics, there was substantial agreement between the two scales of $66 \%(p=0.54)$. The results of both self-report adherence and MPR were also compared with viral load suppression and the difference between viral load suppression and MPR was significant $(p<0.01)$. The difference in adherence between viral load suppression and the self-report measure also showed to be significant $(p<0.01)$.

\section{Introduction}

Adolescents and young people contribute a huge proportion of people living with HIV globally. Generally, young people and adolescents have been more susceptible to HIV infection. By June 2018, it was estimated that, out of the 37.9 million people living with HIV (PLHIV) worldwide, more than $90 \%$ were in the global south, with 1.7 million of them being aged less than 15 years [1]. UNICEF estimated 170,000 new HIV infections among adolescents in 2019 alone[2]. The prevalence of HIV among adolescence in Cameroon was estimated to be $2 \%$ in 2015, with about new 4200 infections and 1900 deaths [3]. As of 2016, a total of about 40,000 adolescents were living with HIV in Cameroon [4].

Globally, adherence has been estimated to be lower in adolescents than in other age groups [5]. A systematic review by Sung-Hee and others in 2014, reported adherence in adolescents to be $62.3 \%$ [5]. In Cameroon self- report adherence to ARVs among adolescents was estimated at $36 \%$ in a study in Yaounde by Fokam and others (Fokam et al., 2017).

All HIV patients are on daily medication and the process is difficult and monotonous such that after six months, adherence to the medications begin to drop as shown in a study by Nsheha and others in 2014 [6]. Given the importance of adherence therefore, it is necessary to take a closer look at adherence and its measurements. This study has hence measured and compared adherence among adolescents using selfreport and Medication Possession Ratio (MPR). 
There are varied methods of measuring adherence with advantages and disadvantages depending on the context and the time[7, 8]. In the absence of directly observed therapy (DOTS), the levels of adherence can only be estimated using other available measures [9]. Some of the available indirect methods of measuring adherence include; self-reports, electronic drug monitoring (EDM), pill counts and pharmacy refill records to obtain medication possession ratio[10]. Adherence can also be measured directly by measuring metabolites including detection of drugs in plasma. However, there is no gold standard of adherence measurement[11] although self-report is the most used tool for adherence measurement[12].

\section{Methods}

\section{Study area and population}

The study was done in some selected health facilities of the North West and South West regions of Cameroon. This study enrolled adolescents (10-19years) who were living with HIV and aware of their status and had been on treatment for at least 6 months.

\section{Study design and Sampling}

The study was designed as an analytical cross-sectional study with a record review component.. The records of the sampled adolescents were reviewed to obtain viral load results. A total of 9 health facilities were purposively selected based on the case load of the number of adolescents registered on treatment. This data was based on information obtained from the HIV Regional Technical Group (RTG) for the North and South West Region. Probability proportionate to size sample allocation was used to obtain the number of adolescents per site. Systematic random sampling was then used select the participants in each of the sampled health facilities and a total of 460 respondents were recruited from the nine health facilities.

\section{Variables and measurements}

For the purpose of this study, adherence was defined as the patient's ability to take medications as per the prescription. Firstly, it was determined through self-report of last missed pills. Self-report adherence was measured based on the 30 days recall. It was calculated as the proportion of pills taken to the number prescribed within 30 days. For example, a patient on one pill daily could have missed 2 pills in the past 30 days, hence the adherence of that participant will be $28 / 30=93 \%$. Based on this premise, participants with a self-report score of $\geq 95 \%$ were considered adherent.

Adherence was also measured through pharmacy records. This calculation from pharmacy records was done based on the Medication Possession ratio (MPR). MPR was calculated as the sum of the days of treatment supplied for all ART prescriptions filled, within the refill interval divided by the number of days during that same time period. A patient was considered as adherent if the MPR was at least $95 \%$. 
Adherence was coded as a binary outcome variable. Only results of respondents who had results for both MPR and self- report were used to compare adherence between the two measures.

Viral load suppression was considered as having the most recent viral load suppression results of less than 1000 copies per $\mathrm{ml}$. The most recent viral load had to be within the past 6 months. Participants with less than 1000 copies/ml were considered having suppressed their viral loads. This viral load was extracted from patient's records in the health facility. All the viral load results done were obtained with the dates on which they were done.

The kappa statistics of inter-rate agreement was used to ascertain the difference between adherence as measured by self-report and MPR. The scale is interpreted as follows (Table 1). Furthermore, the viral load results were also used as a standard to compare adherence as measured by self-report and MPR. The difference in adherence between the two scales were compared using Fischer's exact test and p-values were reported.

Table 1

The Kappa benchmark scale.

\begin{tabular}{|ll|}
\hline Agreement level & Interpretation \\
\hline 0.00 & Poor agreement \\
\hline $0.0-0.20$ & Slight agreement \\
\hline $0.21-0.40$ & Fair agreement \\
\hline $0.41-0.60$ & Moderate agreement \\
\hline $0.61-0.80$ & Substantial agreement \\
\hline $0.81-1.00$ & Almost perfect agreement \\
\hline Source: $[13]$ & \\
\hline
\end{tabular}

\section{Results}

\section{Self- Report ARV Adherence}

As noted earlier, self-report ARV adherence was measured based on a 30-day recall of pills taken. Overall, $336(82.7 \%)$ of the participants were adherent to ARV treatment and the remaining $69(17.0 \%)$ were nonadherent. Hence, the self-report measure of adherence was $83.0 \%$.

\section{Medication Possession Ratio (MPR)}



(73.4\%) of the participants were adherent whereas 111 (26.6\%) of them were non-adherent.

\section{Relationship between self-report and MPR}

Adherence was shown through MPR to be lower than the self- report measure. The relationship between the self-report measure and MPR was assessed. Only the participants who had data for both MPR and self-report were considered. A total of 82 participants had missing data on both MPR and self-report adherence. The Kappa statistics was used to assess the level of agreement between the two measures. The level of agreement was $66 \%(p=0.54)$. Based on the Kappa scale, the $66 \%$ agreement observed indicated that there is substantial agreement between self-report and the medication possession ratio adherence.

Table 2

Relationship between Self-Report adherence measure and Medication Possession Ratio

\begin{tabular}{|llll|}
\hline MPR & & & \\
\hline Self-Report & Adherent N (\%) & Non-Adherent N (\%) & Total \\
\hline Adherent N (\%) & $232(74.8)$ & $78(25.2)$ & 310 \\
\hline Non-adherent N (\%) & $47(74.6)$ & $16(25.4)$ & 63 \\
\hline Total & 279 & 94 & 373 \\
\hline
\end{tabular}

\section{Relationship between Adherence and Viral load}

Having a suppressed viral load may be a strong indication of good adherence. From the analysis, 215 $(61.0 \%)$ of the adolescents who were adherent had a suppressed viral load. The relationship between selfreport and MPR with viral load suppression were assessed. The difference in viral load suppression between those who were adherent for both self-report and MPR and those who were not adherent was significant $(p=0.03)$. Table 3 shows the relationship between viral load suppression and adherence. 
Table 3

Relationship between Viral load suppression and Adherence

\begin{tabular}{|lllc|}
\hline $\begin{array}{l}\text { Type of Adherence } \\
\text { measure }\end{array}$ & $\begin{array}{l}\text { Viral load suppressed } \mathbf{n} \\
(\%)\end{array}$ & $\begin{array}{l}\text { Viral load not suppressed } \mathbf{n} \\
(\%)\end{array}$ & $\begin{array}{l}\boldsymbol{P} \text { - } \\
\text { value }\end{array}$ \\
\hline Self-report & & & - \\
\hline Adherent(Ref) & $215(73.6)$ & $77(26.4)$ & $<0.01$ \\
\hline Non-adherent & $30(52.6)$ & $27(47.4)$ & - \\
\hline MPR & & & $<0.01$ \\
\hline Adherent(Ref) & $189(79.2)$ & $75(20.8)$ & \\
\hline Non-adherent & $25(39.9)$ & $41(60.1)$ & \\
\hline
\end{tabular}

\section{Discussions}

Adherence was also measured through the Medication Possession Ratio (MPR) and the results were lower than the self-report measure. The difference between the two measures was compared using the Kappa statistics. There was substantial agreement of $66 \%$ between the two measures. This suggests that the self-report and MPR method of measuring adherence is likely to yield similar results if used in the same population under same or similar context. Indeed, this study is one of the few studies to compare adherence measurements using self-report and MPR among adolescents and has shown some concordance between the two scales. Earlier studies have compared pill counts and viral load suppression which showed little agreement $(16 \%)$ between the two scales [14]. A study by Denison and others in 2015 had reported adherence using self-report and MPR but did not show the level of agreement between the two methods [15].

Further analysis showed that, only $73.6 \%$ of the adolescents who were adherent (self-report) had suppressed viral load. In the case of MPR, $79.2 \%$ of the adherent adolescents had suppressed viral load. This confirms the trend that adherence to ARV treatment is a main predictor of viral suppression. This result were slightly higher than those of Chikwari in Zimbabwe that reported viral load suppression of $63 \%$ among adherent adolescents [16]. These findings also suggest that MPR may be more predictive of viral load suppression compared to self-report. An earlier study had also shown that pharmacy refill records could suggest HIV virologic failure[17]. The general limitation of the study is the fact that there were missing data as since were some respondents who did not have data for self-report, MPR and viral load suppression and hence could not be included in the final analysis comparing adherence and viral load suppression.

There were also a proportion of adolescents who were non-adherent but had suppressed viral loads. This is possible given that the newer regimens are more forgiving an adherence of $95 \%$ may not be absolutely needed to suppress the virus. 


\section{Conclusion}

Adherence from the self-report measure was higher than from MPR, but there was substantial agreement between the scales. Although there is no gold standard for adherence measurement, self-report or medication possession ratio could be used and complemented with laboratory markers like viral load suppression.

\section{Limitations}

The study reported a proportion of adolescents who were non-adherent but had suppressed viral loads. It has been suggested that this kind of discrepancy is also possible because even in the case of resistance, ARV drugs regimens still exert some degree of anti-HIV activity on the resistant virus [18]. Furthermore, adherence levels between $50-100 \%$ can achieve viral load suppression when using non-nucleoside reverse-transcriptase-inhibitors(NNRTIs) regimens [19].

Findings from the study also revealed that $22 \%$ of adolescents who were adherent to treatment had unsuppressed viral loads. This is counter intuitive as the expectation is that, being adherent should lead to viral load suppression. This could probably be attributed to undiagnosed treatment failure and resistance or the fact that some of the adolescents might have over reported self-report adherence [20]. This is an indication that adherence reports in health settings should be complimented by laboratory markers like viral load to reduce possibilities of unnoticed resistance to treatment which could gradually aggravate to AIDS.

\section{Declarations}

\section{Ethics approval and consent to participate}

Ethical approval was received from the Cameroon Baptist Convention (CBC) ethical review board (IRB2018-41). Administrative approval was obtained from the Regional Delegation of Health at the North and South West Regions of Cameroon

During data collection, written informed consent was obtained from adolescents who were 18years and above and from the guardians of adolescents who were less than 18years. Written assent was then obtained from adolescents less than 18years.

\section{Consent for publication}

Not applicable

\section{Availability of data and materials}


The datasets used and/or analysed during the current study are available as additional files

\section{Funding}

The authors had no funding for this work

\section{Competing interest}

The authors declare no competing interest

\section{Authors' contributions}

$\mathrm{MC}$ and KT was involved in data collection and analysis. KT did the conception and design of the study.MC did the data collection. MC and JG was involved in data analysis and interpretation. AA prepared the draft. All authors read and provided critical inputs to the draft. All authors approved the final version.

\section{Acknowledgements}

Not applicable

\section{References}

1. UNAIDS. Fact sheet - Latest statistics on the status of the AIDS epidemic. 2019.

2. UNICEF. Adolescent HIV Prevention. 2020.

3. UNICEF. Synthesis report of the Rapid Assessment of Adolescent and HIV Programme context in five countries: Botswana, Cameroon, Jamaica, Swaziland and Zimbabwe. All in to End Adolescent AIDS. 2015.

4. UNAIDS. Latest statistics on the status of the AIDS epidemic: UNAIDS; 2017 [updated 2018; cited 2018].

5. Sung-Hee K, Gerver SM, Fidler S, Ward H. Adherence to antiretroviral therapy in adolescents living with HIV: systematic review and meta-analysis. Aids. 2014;28(13):1945-56.

6. Nsheha AH, Dow DE, Kapanda GE, Hamel BC, Msuya LJ. Adherence to antiretroviral therapy among HIV-infected children receiving care at Kilimanjaro Christian Medical Centre (KCMC), Northern Tanzania: A cross-sectional analytical study. Pan African Medical Journal. 2014;17(1).

7. Castillo-Mancilla JR, Haberer JE. Adherence measurements in HIV: new advancements in pharmacologic methods and real-time monitoring. Current Hiv/aids Reports. 2018;15[1]:49-59. 
8. Evans D, Fox MP. When and how should we be measuring adherence to antiretroviral therapy in resource-limited settings. J Clin Res HIV AIDS Prev. 2013;1(2):24-30.

9. Gill CJ, Hamer DH, Simon JL, Thea DM, Sabin LL. No room for complacency about adherence to antiretroviral therapy in sub-Saharan Africa. Aids. 2005;19[12]:1243-9.

10. Berg KM, Arnsten JH. Practical and conceptual challenges in measuring antiretroviral adherence. Journal of acquired immune deficiency syndromes (1999). 2006;43(Suppl 1):S79.

11. Zhang Q, Li X, Qiao S, Shen Z, Zhou Y. Comparing self-reported medication adherence measures with hair antiretroviral concentration among people living with HIV in Guangxi, China. AIDS Research and Therapy. 2020;17(1):1-8.

12. Chesney MA. The elusive gold standard: future perspectives for HIV adherence assessment and intervention. JAIDS Journal of Acquired Immune Deficiency Syndromes. 2006;43:S149-S55.

13. Klein D, editor Assessing inter-rater agreement in Stata. German Stata Users' Group Meetings; 2017.

14. Martelli G, Antonucci R, Mukurasi A, Zepherine H, Nöstlinger C. Adherence to antiretroviral treatment among children and adolescents in Tanzania: Comparison between pill count and viral load outcomes in a rural context of Mwanza region. PloS one. 2019;14[3]:e0214014.

15. Denison JA, Koole O, Tsui S, Menten J, Torpey K, Van Praag E, et al. Incomplete adherence among treatment-experienced adults on antiretroviral therapy in Tanzania, Uganda and Zambia. AIDS (London, England). 2015;29(3):361.

16. Chikwari CD, Ferrand RA, Simms V. Association between self-reported adherence and HIV viral load suppression among older children and adolescents. Journal of acquired immune deficiency syndromes (1999). 2017;76(3):e87.

17. Gaifer Z, Boulassel M-R. Comparative Analysis of two Methods of Measuring Antiretroviral Therapy Adherence in HIV-Infected Omani Patients. Journal of the International Association of Providers of AIDS Care (JIAPAC). 2019;18:2325958219867316.

18. Bangsberg DR, Deeks SG. Is average adherence to HIV antiretroviral therapy enough? Journal of general internal medicine. 2002;17(10):812.

19. Bangsberg DR. Less than $95 \%$ adherence to nonnucleoside reverse-transcriptase inhibitor therapy can lead to viral suppression. Clinical infectious diseases. 2006;43(7):939-41.

20. Cruz ML, Cardoso CA, Darmont MQ, Souza E, Andrade SD, D'Al Fabbro MM, et al. Viral suppression and adherence among HIV-infected children and adolescents on antiretroviral therapy: results of a multicenter study. Jornal de pediatria. 2014;90(6):563-71.

\section{Supplementary Files}

This is a list of supplementary files associated with this preprint. Click to download.

- Datasetadherencemeasurementsinadolescents.xlsx

- Datasetadherencemeasurementsinadolescents.xlsx 